\title{
Review Article: On the relation between the seismic activity and the Hurst exponent of the geomagnetic field at the time of the 2000 Izu swarm
}

\author{
F. Masci $^{1}$ and J. N. Thomas ${ }^{2,3,4}$ \\ ${ }^{1}$ Istituto Nazionale di Geofisica e Vulcanologia, L'Aquila, Italy \\ ${ }^{2}$ Northwest Research Associates, Redmond, Washington, USA \\ ${ }^{3}$ Department of Electrical and Computer Engineering, Digipen Institute of Technology, Redmond, Washington, USA \\ ${ }^{4}$ Department of Earth and Space Sciences, University of Washington, Seattle, Washington, USA \\ Correspondence to: F. Masci (fabrizio.masci@ingv.it)
}

Received: 28 January 2013 - Published in Nat. Hazards Earth Syst. Sci. Discuss.: 26 March 2013

Revised: 7 June 2013 - Accepted: 21 July 2013 - Published: 6 September 2013

\begin{abstract}
Many papers document the observation of earthquake-related precursory signatures in geomagnetic field data. However, the significance of these findings is ambiguous because the authors did not adequately take into account that these signals could have been generated by other sources, and the seismogenic origin of these signals have not been validated by comparison with independent datasets. Thus, they are not reliable examples of magnetic disturbances induced by the seismic activity. Hayakawa et al. (2004) claim that at the time of the 2000 Izu swarm the Hurst exponent of the Ultra-Low-Frequency (ULF: $0.001-10 \mathrm{~Hz}$ ) band of the geomagnetic field varied in accord with the energy released by the seismicity. The present paper demonstrates that the behaviour of the Hurst exponent was insufficiently investigated and also misinterpreted by the authors. We clearly show that during the Izu swarm the changes of the Hurst exponent were strongly related to the level of global geomagnetic activity and not to the increase of the local seismic activity.
\end{abstract}

\section{Introduction}

Several researchers consider the investigation of the geomagnetic field in the Ultra-Low-Frequency (ULF) band as a useful tool for monitoring possible earthquake-related magnetic signatures, as well as precursory signals. Despite many reports of magnetic ULF earthquake precursors, some re- searchers do not agree that these are actually earthquake related. According to them, the observed magnetic anomalies were generated by other sources, such as global geomagnetic activity (see e.g. Campbell, 2009; Masci, 2010; Thomas et al., 2009a), or they were caused by instrument malfunction (see e.g. Masci, 2012b; Thomas et al., 2009b). These studies led to a re-examination of many controversial observations of earthquake-related signals, which found that several methodologies used to identify magnetic precursors were invalid. Among these methods are magnetic polarization ratio (see Thomas et al., 2009b; Masci, 2011a, 2012a, c), fractal characteristics of the geomagnetic field components (see Masci, 2010, 2013a; Masci and Thomas, 2013), and eigenvalues of the principal component analysis (see Masci, 2011b). Reports of ionospheric earthquake-related disturbances were also investigated (see Masci, 2012d, 2013b; Thomas et al., 2012). Bearing in mind these findings, the reliability of earthquake precursory signals must be carefully investigated by means of independent data.

At the end of June 2000, a seismic swarm started offshore of the Izu peninsula, Japan. Five strong $(M>6)$ earthquakes occurred on 1, 8, 15, and 30 July and 18 August. Several papers, with different methodologies, such as fractal analysis, polarization ratio analysis, and principal component analysis document the observation of presumed seismogenic signals that occurred before and during the swarm (see Gotoh et al., 2003, 2004; Ismaguilov et al., 2003; Hayakawa, 2011). However, these reports did not show that the geomagnetic 
anomalies and seismic activity were physically related. On the contrary, recent studies by Masci (2010, 2011a, b) clearly demonstrated that before and during the 2000 Izu swarm the presumed seismogenic signatures in the magnetic field were just normal signals highly correlated to global geomagnetic activity caused by solar wind-magnetosphere and magnetosphere-ionosphere interactions.

Here, we investigate the findings by Hayakawa et al. (2004) that at the time of the 2000 Izu swarm the statistical proprieties of the ULF component of the geomagnetic field were strongly correlated with the energy released by the seismic activity.

\section{Discussion}

Hayakawa et al. (2004), in a report published by Physics and Chemistry of the Earth, claim that at the time of the 2000 Izu swarm the Hurst exponent of the ULF band of the geomagnetic field varied with local seismic activity. They analyse data from the geomagnetic station of Seikoshi (sampling rate $1 \mathrm{~Hz})$ and from the seismic station of Mohikoshi. The two stations are about $80 \mathrm{~km}$ away from the epicentral area. See Appendix A for a brief discussion on the Hurst exponent and how to calculate it for a discrete time series.

Hayakawa et al. (2004) used principal component analysis to find the trend of time variation of the Hurst exponent of the geomagnetic field during 2000. Principal component analysis is a method that converts data into a set of uncorrelated variables called principal components (Jolliffe, 2002). Hayakawa et al. (2004) investigated the behaviour of the 1st principal component of the Hurst exponent of the geomagnetic field. Simply speaking, the 1st principal component can be regarded as a trend of a time series. In the following discussion we define a Hurst exponent as the 1st principal component. According to Hayakawa et al. (2004), the local seismic activity and the Hurst exponent of the geomagnetic field in the ULF frequency band show a strong correlation from the end of June to the first weeks of July 2000. Figure 1 shows the behaviour from February to December 2000 of the Hurst exponent of the geomagnetic field $H$ component $(H u-H)$ and local seismic activity $\left(M^{*}\right)$ as reported by Hayakawa et al. (2004). $M^{*}$ is the earthquake magnitude estimated by the Japan Meteorological Agency. Yellow-bounded areas highlight the period during which $H u-H$ seems to vary in accord with $M^{*}$. Our first observation concerns the lack of a corresponding correlation after the middle of July 2000 when, as can be seen from Fig. 1, the seismic swarm was still in progress. This casts some doubt on the possible relationship between $H u-H$ and $M^{*}$. Hayakawa et al. (2004) noted that $M^{*}$ and $H u-H$ do not vary coherently all the time. The authors justify the lack of correlation after the middle of July invoking "a kind of saturation" that took place during the evolution of the seismic swarm. Unfortunately, they do not explain the true meaning of the supposed "saturation", nor they specify what saturated. (a) $\mathrm{H}$ - field component

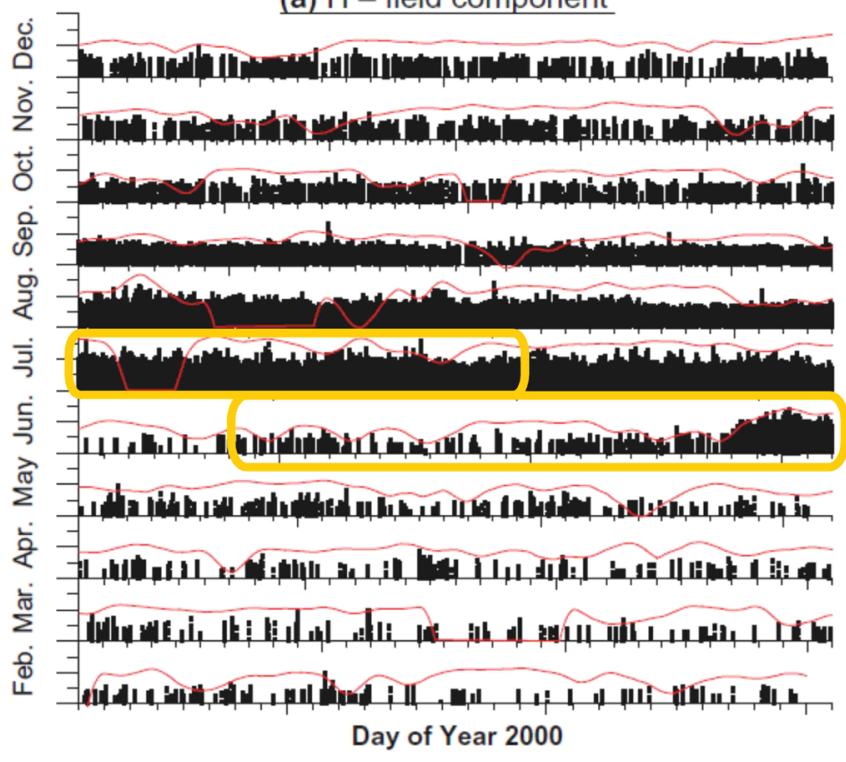

Fig. 1. A reproduction of Fig. 1 by Hayakawa et al. (2004). The vertical black bars represent the earthquake magnitude $M^{*}$ estimated by the Japan Meteorological Agency, whereas the solid red line represents the behaviour of the Hurst exponent of the geomagnetic field $H$ component during 2000. Yellow rounded rectangles highlight the period in which the Hurst exponent is claimed to change coherently with $M^{*}$ by Hayakawa et al. (2004).

Figure 2 is a reproduction of Fig. 2 by Hayakawa et al. (2004). The figure shows the local seismic activity $\left(M^{*}\right)$ and the Hurst exponent of the geomagnetic field components $H$, $D$, and $Z$ during the period from February to August 2000. Enlarged views from 7 June to 18 July shows both daily values and \pm 3 -day running average of the Hurst exponent compared with the \pm 3 -day running average of the earthquake magnitude $M^{*}$. According to Hayakawa et al. (2004), there is a strong correspondence between the increase of $M^{*}$ and the variation of the Hurst exponent of the geomagnetic field horizontal components $H$ and $D$. Conversely, the Hurst exponent of the vertical component $Z$ of the geomagnetic field does not show a similar pronounced correspondence with the seismic activity.

Considering that interaction of the solar wind with the Earth's magnetosphere and ionosphere-magnetosphere coupling are the main sources of ULF disturbances (see McPherron, 2005; Saito, 1969), we compare the findings of Hayakawa et al. (2004) with global geomagnetic activity by means of the $\Sigma \mathrm{Kp}$ index time series. The $\Sigma \mathrm{Kp}$ index is calculated by magnetic observations from a planetary network of 13 geomagnetic observatories. Possible magnetic pre-earthquake anomalies of tectonic origin are local signals having relatively weak intensity. Consequently, the $\Sigma \mathrm{Kp}$ index is not influenced by possible crustal signals, otherwise, these signals should be observed in most of the 13 magnetic observatories. 


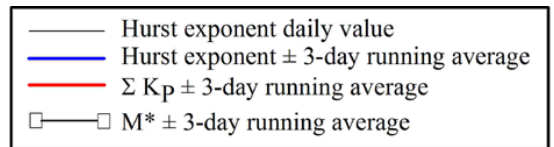

(a) $\quad H$-field component (the meridian plane)

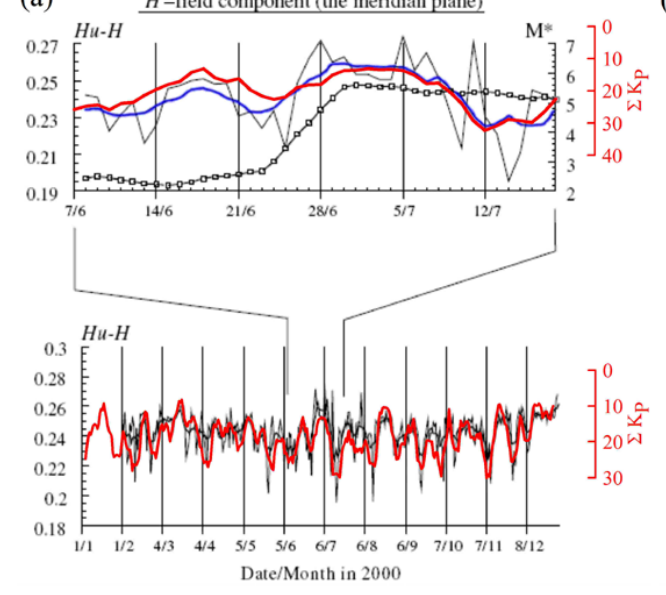

(c) $\underline{Z}$-field component (the vertical plane)

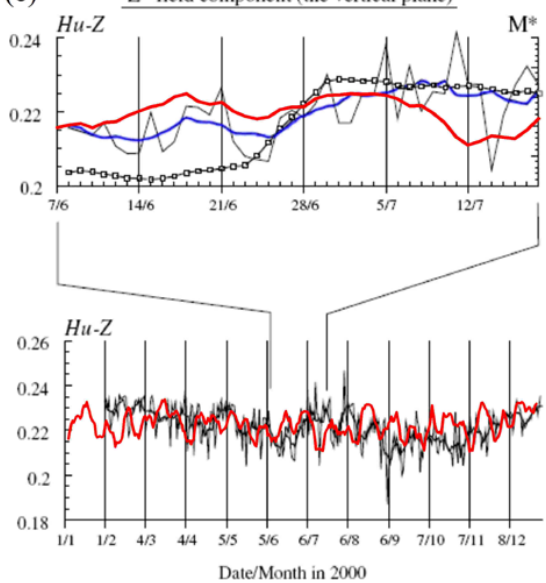

(b) $\quad \underline{D \text {-field component (across the meridian) }}$
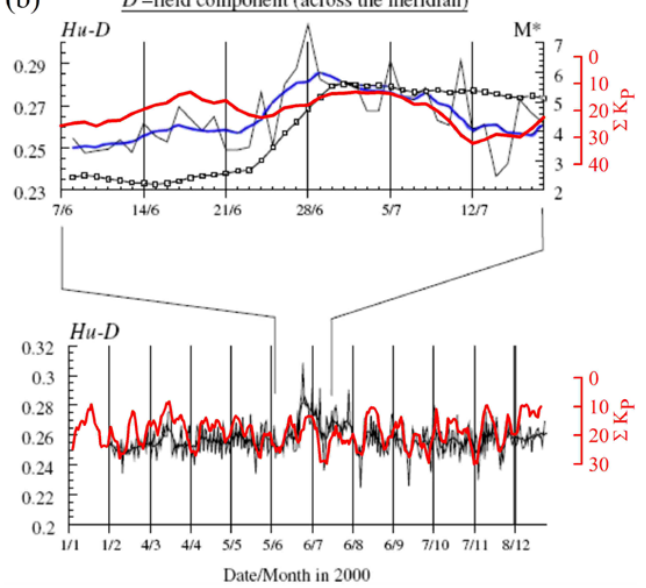

(d) Daly averaged EQ JMA magnitude $\mathrm{M}^{*}$

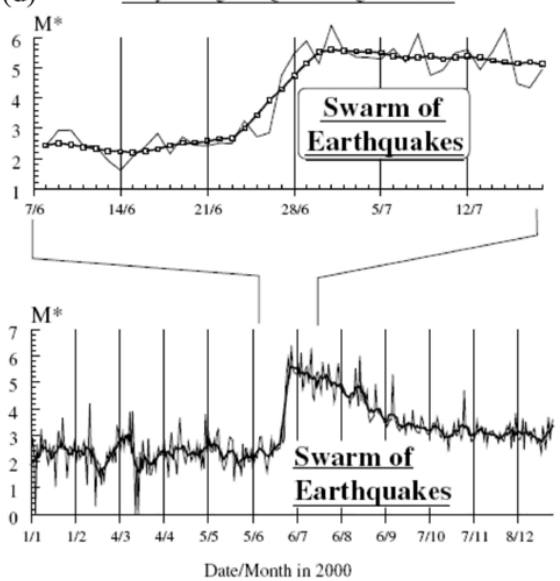

Fig. 2. A reproduction of Fig. 2 by Hayakawa et al. (2004). (a), (b), and (c) the variation of the Hurst exponent (daily values and \pm 3 -day running average) of the geomagnetic field components $H, D$, and $Z$ during 2000. The enlarged views of each panel refer to the rise of the seismic activity at Izu. (d) daily values and \pm 3 -day running average of the earthquake magnitude $M^{*}$. The \pm 3 -day running average of $M^{*}$ (solid line with open squares) is also reported in panels (a), (b), and (c). The \pm 3 -day running average of the global geomagnetic index $\Sigma \mathrm{Kp}$ is superimposed on the original views. See text for details.

In Fig. 2, the \pm 3 -day running average of the $\Sigma \mathrm{Kp}$ index is superimposed onto the original views by Hayakawa et al. (2004). We found a strong correlation between the \pm 3 -day running averages of the Hurst exponent of the horizontal geomagnetic field and $\Sigma \mathrm{Kp}$ on both short and long timescales. Namely, the Hurst exponent shows a close correlation with $\Sigma \mathrm{Kp}$ during the entire period of time (February-December 2000) reported in the figure and not only during few weeks from 7 June to 18 July. This correlation is particularly evident in the $H$ component. This fact suggests that the variations of the Hurst exponent of the geomagnetic field are closely related to changes in geomagnetic activity. In addition, the greater correspondence with the geomagnetic index of the Hurst exponent of the horizontal components $H$ and $D$ is clearly justified because the $\mathrm{Kp}$ index is calculated using these components of the geomagnetic field (Mayaud, 1980).

Figure 3 shows in detail the comparison between $M^{*}$, $\Sigma \mathrm{Kp}$, and the Hurst exponent of the geomagnetic field $H$ component $(H u-H)$, during the period from 7 June to 18 July 2000 . The daily values and the \pm 3 -day running averages of $H u-H$ and $M^{*}$ were obtained by digitizing the original view of Hayakawa et al. (2004). We can see the strong inverse correlation that exists between the daily values of $H u-H$ and $\Sigma \mathrm{Kp}$. Figure $3 \mathrm{~b}$ and c show the linear relationships between the \pm 3 -day running averages of $H u-H$ and $\Sigma \mathrm{Kp}$, and $H u-H$ and $M^{*}$, respectively. It is evident that the strong correlation that exists between $H u-H$ and $\Sigma \mathrm{Kp}$ (correlation coefficient $=-0.87$ ) cannot be stated for the pair $H u-H$ and $M^{*}$ 

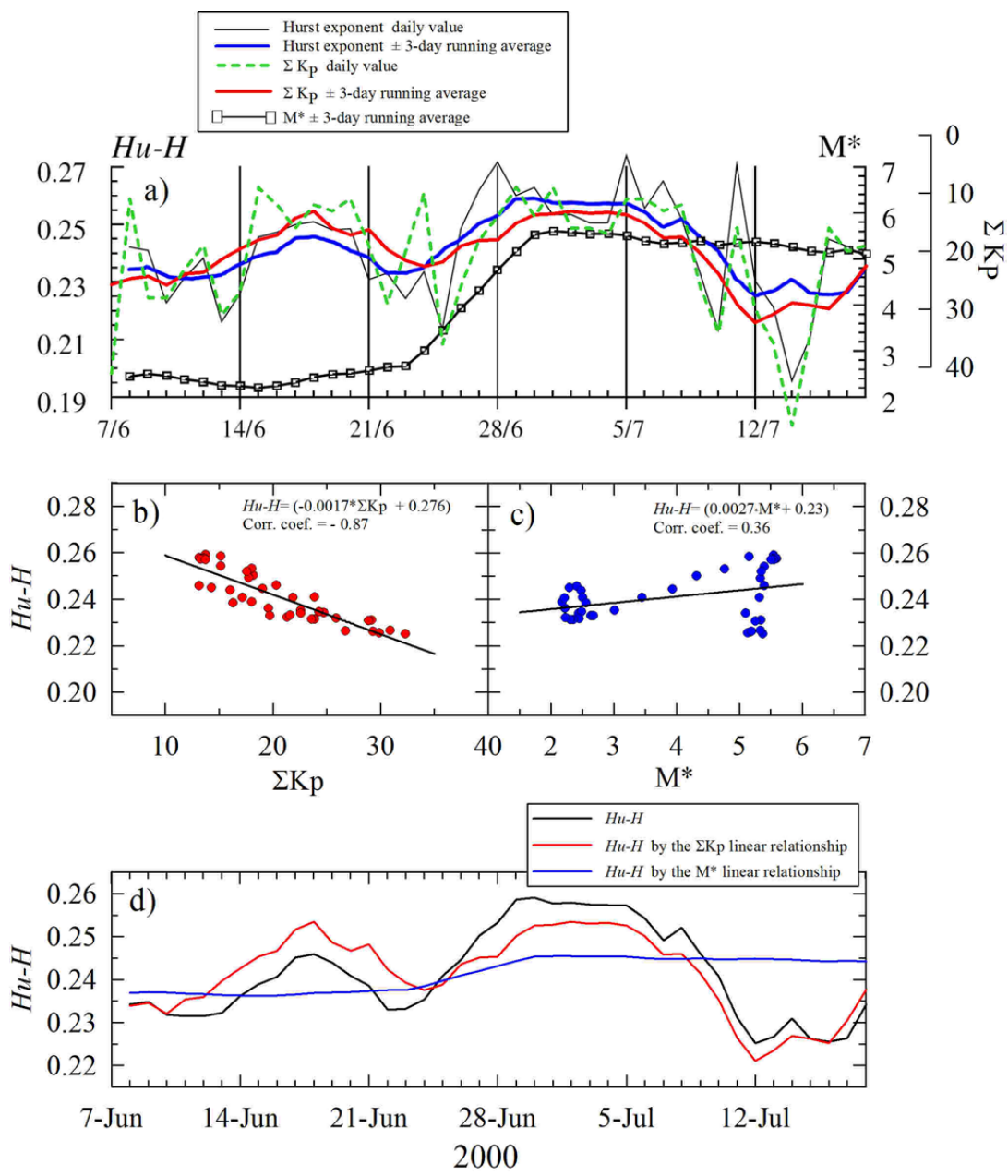

Fig. 3. (a) A reproduction of Fig. 2 by Hayakawa et al. (2004). Variation of the Hurst exponent (daily values and \pm 3 -day running average) of the geomagnetic field $H$ component at the time of the Izu swarm. The \pm 3 -day running average of earthquake magnitude $M^{*}$ (solid line with open squares) is also shown. $\Sigma \mathrm{Kp}$ time series (daily values and \pm 3 -day running average) have been superimposed onto the original view. (b) and (c) linear relationships between the \pm 3 -day running average of the Hurst exponent and $\Sigma \mathrm{Kp}$ and $M^{*}$, respectively. (d) the Hurst exponent calculated by Hayakawa et al. (2004) compared with reconstructed Hurst exponents using the linear relationships shown in panels (b) and (c). See text for details.

(correlation coefficient $=0.36$ ). This finding is confirmed by Fig. $3 \mathrm{~d}$ that shows the original $H u-H$ time series and the $H u-H$ time series reconstructed using the linear relationships with $\Sigma \mathrm{Kp}$ and $M^{*}$, respectively. We can see that $H u-H$ constructed by the linear relationship with $\Sigma \mathrm{Kp}$ is very similar to the original $\mathrm{Hu}-\mathrm{H}$ time series. On the contrary, this cannot be stated for the $\mathrm{Hu}-\mathrm{H}$ time series constructed by the $\mathrm{M}^{*}$ linear relationship.

We note that during the period of 23-29 June 2003 there is a correspondence between the increases of $H u-H$ and $M^{*}$, but in our opinion this is just a chance event that led the authors to believe that the behaviour of the Hurst exponent was influenced by possible magnetic signals induced by seismicity. The real issue is the lack of correlation between $\mathrm{Hu}-\mathrm{H}$ and $M^{*}$ after 29 June (and also in the following months) when the swarm was still in progress. On the contrary, as previously stated, $H u-H$ has a strong correlation with the geomagnetic activity level $(\Sigma \mathrm{Kp})$ during the entire period of time, from February to December 2000, shown by Hayakawa et al. (2004). This clearly demonstrates that the variation of the Hurst exponent of the geomagnetic field was induced by geomagnetic activity changes and not by seismicity.

\section{Conclusions}

Here we have shown that variation of the Hurst exponent of the ULF geomagnetic field during February to December 2000, and particularly from the beginning of June to the middle of July, is highly correlated to geomagnetic activity. Moreover, contrary to the claims of Hayakawa et al. (2004), we have found that during the 2000 Izu swarm the behaviour 
of the Hurst exponent of the ULF geomagnetic field is poorly correlated with the energy released by the local seismic activity. This paper is a further confirmation of the findings of Masci (2010, 2011a, b), which demonstrated that many presumed magnetic seismogenic signatures claimed to be related to the Izu swarm of 2000 were actually normal magnetospheric disturbances.

\section{Appendix A}

The Hurst exponent, also called "index of long-range dependence", is a measure of long-term memory of a time series. It quantifies the tendency of a time series either to regress to the mean value, or to cluster in a direction. The Hurst exponent was introduced in the analysis of hydrological data by Hurst (1951).

Hurst introduced empirically the concept of rescaledrange analysis. Given a discrete time series $X$ of length $N$, we can divide it into subseries $x_{n}$ of length $n=N, N / 2$, $N / 4, \ldots$. For each series $x_{n}$ of length $n$ we calculate the mean $\bar{x}_{n}$ and the standard deviation $S_{n}$

$\bar{x}_{n}=\frac{1}{n} \sum_{i=1}^{n} x_{i}, \quad S_{n}=\sqrt{\frac{1}{n-1} \sum_{i=1}^{n}\left(x_{i}-\bar{x}_{n}\right)^{2}}$.

We now create, for $j=1,2, \ldots, n$, the series of differences from the mean $y_{j}=x_{j}-\bar{x}_{n}$ and we define the cumulative deviate series from the mean

$Y_{j}=\sum_{i=1}^{j} y_{i}$

The range $R_{n}$ is defined by

$R_{n}=\left(Y_{j}\right)_{\max }-\left(Y_{j}\right)_{\min }$,

i.e. the difference between the maximum and minimum values of $Y_{j}$.

The so-called rescaled-range of the subseries $x_{n}$ is defined by the ratio $R_{n} / S_{n}$. Hurst found that $R_{n} / S_{n}$ scales, with respect to the length $n$ of the subseries $x_{n}$, by the power law

$R_{n} / S_{n} \propto n^{H u}$.

The exponent $H u$ is then estimated by the slope of the bestfit line in $\log \left(R_{n} / S_{n}\right)$ versus $\log (n)$ representation of the $R_{n} / S_{n}$ power law.

Hurst found empirically that many time series of natural phenomena are well described by the previous relation in which $H u$ is known as the Hurst exponent. $H u$ assume values in the range $0<H u<1$. A value of $H u>0.5$ indicates a time series with a long-term positive autocorrelation (persistent behaviour), i.e. if the time series increases in a time interval it continues to increase in the following interval. Simply speaking, the past influences the future. For $H u<0.5$ there is a negative autocorrelation between the values of a time series (anti-persistent behaviour), i.e. if the time series increases in a time interval it is likely that it decrease in the following interval. $H u=0.5$ indicates a completely uncorrelated time series.

Acknowledgements. The authors wish to thank the Editor and three anonymous referees for their comments that were useful for improving the manuscript. We also thank the World Data Center for Geomagnetism, Kyoto University for providing Kp index. JNT was partially supported by the USGS Earthquake Hazards Program, external research grant G11AP20177.

Edited by: B. D. Malamud

Reviewed by: three anonymous referees

\section{References}

Campbell, W. H.: Natural magnetic disturbance fields, not precursors, preceding the Loma Prieta earthquake, J. Geophys. Res., 114, A05307, doi:10.1029/2008JA013932, 2009.

Gotoh, K., Hayakawa, M., and Smirnova, N.: Fractal analysis of the ULF geomagnetic data obtained at Izu Peninsula, Japan in relation to the nearby earthquake swarm of June-August 2000, Nat. Hazards Earth Syst. Sci., 3, 229-236, doi:10.5194/nhess-3229-2003, 2003.

Gotoh, K., Hayakawa, M., and Smirnova, N. A., Hattori, K.: Fractal analysis of seismogenic ULF emissions, Phys. Chem. Earth, 29, 419-424, doi:10.1016/j.pce.2003.11.013, 2004.

Hayakawa, M.: On the fluctuation spectra of seismoelectromagnetic phenomena, Nat. Hazards Earth Syst. Sci., 11, 301-308, doi:10.5194/nhess-11-301-2011, 2011.

Hayakawa, M., Hattori, K., Nickolaenko, A. P., and Rabinowicz, L. M.: Relation between the energy of earthquake swarm and the Hurst exponent of random variations of the geomagnetic field, Phys. Chem. Earth, 29, 379-387, doi:10.1016/j.pce.2003.07.001, 2004.

Hurst, H. E.: Long-term storage capacity of reservoirs, T. Am. Soc. Civ. Eng., 116, 770-808, 1951.

Ismaguilov, V. S., Kopytenko, Yu. A., Hattori, K., and Hayakawa, M.: Variations of phase velocity and gradient values of ULF geomagnetic disturbances connected with the Izu strong earthquakes, Nat. Hazards Earth Syst. Sci., 3, 211-215, doi:10.5194/nhess-3-211-2003, 2003.

Jolliffe, I. T.: Principal Component Analysis, Springer-Verlag, 2nd Edn., 487 pp., 2002.

Masci, F.: On claimed ULF seismogenic fractal signatures in the geomagnetic field, J. Geophys. Res., 115, A10236, doi:10.1029/2010JA015311, 2010.

Masci, F.: On the seismogenic increase of the ratio of the ULF geomagnetic field components, Phys. Earth Planet. In., 187, 19-32, doi:10.1016/j.pepi.2011.05.001, 2011a.

Masci, F.: Brief communication "On the recent reaffirmation of ULF magnetic earthquakes precursors”, Nat. Hazards Earth Syst. Sci., 11, 2193-2198, doi:10.5194/nhess-11-2193-2011, 2011 b.

Masci, F.: Comment on "Ultra Low Frequency (ULF) European multi station magnetic field analysis before and during the 2009 
earthquake at L'Aquila regarding regional geotechnical information” by Prattes et al. (2011), Nat. Hazards Earth Syst. Sci., 12, 1717-1719, doi:10.5194/nhess-12-1717-2012, 2012a.

Masci, F.: Comment on "Possible association between anomalous geomagnetic variations and the Molise Earthquakes at Central Italy during 2002" by Takla et al. (2011), Phys. Earth Planet. In., 202-203, 92-94, doi:10.1016/j.pepi.2012.02.006, 2012b.

Masci, F.: On the ULF magnetic ratio increase before the 2008 Iwate-Miyagi Nairiku earthquake by Hirano and Hattori (2011), J. Asian Earth Sci., 56, 258-262, doi:10.1016/j.jseaes.2012.05.020, 2012c.

Masci, F.: The study of ionospheric anomalies in Japan area during $1998-2010$ by Kon et al.: An inaccurate claim of earthquake- related signatures?, J. Asian Earth Sci., 57, 1-5, doi:10.1016/j.jseaes.2012.06.009, 2012d.

Masci, F.: On the multi-fractal characteristics of the ULF geomagnetic field before the 1993 Guam earthquake, Nat. Hazards Earth Syst. Sci., 13, 187-191, doi:10.5194/nhess-13-187-2013, 2013a.

Masci, F.: Brief communication "Further comments on the ionospheric precursor of the 1999 Hector Mine earthquake", Nat. Hazards Earth Syst. Sci., 13, 193-196, doi:10.5194/nhess-13193-2013, 2013b.
Masci, F. and Thomas, J. N.: Comment on "Fractal analysis of ULF electromagnetic emissions in possible association with earthquakes in China” by Ida et al. (2012), Nonlin. Processes Geophys., 20, 417-421, doi:10.5194/npg-20-417-2013, 2013.

Mayaud, P. N.: Derivation, Meaning, and Use of Geomagnetic Indices,American Geophysical Union, Washington, D. C., Geoph. Monog. Series, 22, 154 pp., doi:10.1029/GM022, 1980.

McPherron, R. L.: Magnetic pulsations: their sources and relation to solar wind and geomagnetic activity, Surv. Geophys., 26, 545592, doi:10.1007/s10712-005-1758-7, 2005.

Saito, T.: Geomagnetic pulsations, Space Sci. Rev., 10, 319-342, doi:10.1007/BF00203620, 1969.

Thomas, J. N., Love, J. J., and Johnston, M. J. S.: On the reported magnetic precursor of the 1989 Loma Prieta earthquakes, Phys. Earth Planet. In., 173, 207-215, doi:10.1016/j.pepi.2008.11.014, 2009a.

Thomas, J. N., Love, J. J, Johnston, M. J. S., Yumoto, K.: On the reported magnetic precursor of the 1993 Guam earthquake, Geophys. Res. Lett., 36, L16301, doi:10.1029/2009GL039020, 2009b.

Thomas, J. N., Love, J. J., Komjathy, A., Verkhoglyadova, O. P., Butala, M., and Rivera, N.: On the reported ionospheric precursor of the 1999 Hector Mine, California earthquake, Geophys. Res. Lett., 39, L06302, doi:10.1029/2012GL051022, 2012. 